\title{
Preformulation Considerations Development and Evaluation of Mesalamine Loaded Polysaccharide-Based Complex Mucoadhesive Beads for Colon Targeting
}

\author{
Rohitas Deshmukh*, Ranjit Kumar Harwansh \\ Institute of Pharmaceutical Research, GLA University, Mathura, Uttar Pradesh, INDIA.
}

\begin{abstract}
Background: Inflammatory bowel disease (IBD) is a chronic inflammatory disease of large intestine consisting of ulcerative colitis and Crohn's disease. Mesalamine is a first line drug use for the treatment but it has drawback of low water solubility and low permeability. In addition, oral drug delivery to colon faces the problem of gastric degradation and thus increases dose size and frequency. Objectives: The present study was aimed to assay mesalamine and develop an enteric coated mesalamine loaded mucoadhesive beads using guar gum, sodium alginate and carbopol 940 for an effective delivery to colon. Methods: Preformulation study was done to check the purity of drug. The beads were prepared by ion gelation methods and coated with Eudragit S100 and characterized. Results: The melting point and absorbance maxima of mesalamine were found to be $282^{\circ} \mathrm{C}$ and $220 \mathrm{~nm}$ respectively. The FTIR study confirms the drug and polymers were compatible. A total 5 different formulations were prepared which specifically release the drug in the colon region in sustain release fashion. The formulation MA-F4 was chosen as an optimized formulation. The Particle size, Zeta potential, \%EE, \%Yield, \%DL and in vitro drug release of optimized formulation was found to be $445.6 \pm 67.1 \mu \mathrm{m},-28.01$ $\pm 0.16 \mathrm{mV}, 94.98 \pm 3.22,96.27 \pm 3.22,30.4 \pm 0.9$ and $95.07 \pm 3.85$ respectively. Conclusion: The study demonstrated that the prepared beads can release the mesalamine in sustained release manner and helps in management of IBD.
\end{abstract}

Key words: Inflammatory bowel disease, Colon, Mesalamine, Mucoadhesion, Beads.

\section{INTRODUCTION}

Inflammatory Bowel Disease (IBD) is a multidimensional gastrointestinal (GIT) disease. IBD involves major 2 types of inflammatory problems such as Crohn's disease (CD) and ulcerative colitis (UC) which may be either progressive or chronic with unknown etiology. ${ }^{1-3}$ The intestinal clinical symptoms associated with UC and $\mathrm{CD}$ are abdominal pain, bloody diarrhea, weight loss and vomiting whereas extraintestinally are mostly affected with eyes, joints, skin and bile duct. IBD have been associated with numerous factors like environment, immune system, microorganism, GIT microflora and genes. IBD pathogenesis and development is also linked with irregular protein glycosylation., ${ }^{2,4}$ If these problems is not early diagnosed and treated, severe intestinal complications can be arisen in the form of fistulae, extreme abdominal pain, colon cancer, bowel function impairment and disability. ${ }^{5,6}$ Till date a permanent treatment of IBD is not established therefore the treatment of disease is symptomatic and sometime lifelong. Globally, the occurrence and morbidity of IBD is dramatically growing and influence all ages of population including pediatric and geriatric patients and affect their overall life., ${ }^{7,8}$ Currently, epidemiological survey revealed that incidence of IBD
Submission Date: 07-08-2020; Revision Date: 02-12-2020; Accepted Date: 20-01-2021

DOI: 10.5530/ijper.55.1.13 Correspondence: Dr. Rohitas Deshmukh Institute of Pharmaceutical Research, GLA University, Mathura-281406, Uttar Pradesh, INDIA Phone no: +917987367129 Email id: rahi18rahi@gmail. com

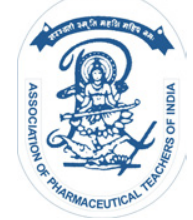

www.ijper.org 
in developing countries like India is rising which is challengeable task. ${ }^{9}$

An anti-inflammatory drug like 5-amino salicylates (5-ASA) is the first line therapy used for UC and CD. It is safe and well tolerated in most of the cases. 5-ASA drugs, like Sulfasalazine is the first choice drug for the treatment of IBD, mesalamine is another choice of drug used in the treatment of mild to moderate conditions of IBD. ${ }^{10,11}$ Corticosteroids, like prednisolone and budesonide are used when aminosalicilates does not give desired results. These drugs are used for management of moderate to severe IBD. ${ }^{12}$ Immunomodulators agents, like 6-mercaptopurine, azathioprine and methotrexate, antibiotics like metronidazole, rifaximin, ciprofloxacin and most important anti-TNF- $\alpha$-antibodies (infliximab, adalimumab, golimumab and certolizumab) also play a significant role in the management of severe disease stages., ${ }^{313-15}$ Mesalamine is a first line drug for the treatment of mild to moderate IBDs including UC and CD. ${ }^{16}$ Patients with IBD exhibit an increased generation of leukotrienes and hydroxy eicosatetraenoic acids through cyclooxygenase (COX) and lipoxygenase (LOX) pathways. It is assumed that mesalamine block the cyclooxygenase and inhibit the production of prostaglandin and thus diminishing the inflammation in the colon. ${ }^{17}$ It is $\mathrm{pH}$-dependent drug and can release in the environment of colon, where maximum concentration of drug can be achieved at targeted site. ${ }^{11,18}$

Mesalamine belongs to class IV of BCS which means it has low water solubility and low permeability thus low bioavailability in the cells. This drawback of mesalamine is a major challenge for the formulator to design a suitable delivery system. Targeted oral drug delivery system is pioneering for improving drug efficacy and bioavailability at the site of action with mucoadhesive approach. ${ }^{19}$ In the last few decades, a continuous quest has been raised towards colonic delivery of drugs to enhance their therapeutic efficacy at target sites and minimize related side effects. Although, merely a few approaches like colon targeted drug delivery system that has the ability to work in a complex environment of the gastrointestinal tract (GIT) of the human. The polymeric microparticles prepared from polysaccharides are attractive carrier system for colon targeting because of their properties like $\mathrm{pH}$ swellability in specific $\mathrm{pH}$ and degradability in colonic lumen by bacteria. ${ }^{20,21}$ Thus, mucoadhesive drug delivery system is highly required for site specific treatment for IBD which would be fulfilled by micro-particulate carrier system.

Guar gum (GG) is natural gum which has been well explored as a polymer for colon targeting due to its extraordinary gelling efficiency, mucoadhesiveness, biodegradability and sustained release property. ${ }^{22}$ Sodium alginate $(\mathrm{SA})$ is a anionic polysaccharide, alginic acid with sodium salt, obtained from seaweeds. The specific feature of SA includes mucoadhesiveness, $\mathrm{pH}$-sensitivity, cross-linking capability, low toxicity and biodegradability that offer more suitability for colon drug targeting. ${ }^{23}$ Carbopol 940 (CP) is a high molar mass cross-linked polyacrylic acid polymer having extremely wetting and colloidal viscosity property (1000 times more viscous) which forms a sparkling clear gel in water. ${ }^{24}$

Present study was aimed to assay physiochemical properties of mesalamine and to develop mesalamine loaded mucoadhesive microcarrier for an effective delivery to colon against IBD. The microparticles were coated with a Eudragit S 100 (enteric coating polymer) which dissolve specifically at colonic $\mathrm{pH}$ (7.4) and allow the drug to release in the colon region in sustain release fashion. Thus, after oral administration, enteric coated microparticles will be presented in unchanged form in stomach environment but it will be released in alkaline $\mathrm{pH}$ of colonic fluid which may be suitable for management of IBD.

\section{MATERIALS AND METHODS}

\section{Materials}

Mesalamine standard drug was purchased from Yarrow chem, Mumbai, India. Sodium alginate, Guar gum, Eudragit S-100 and carbopol 940 were purchased from $\mathrm{CDH}$, New Delhi, India. Calcium chloride dihydrate were obtained from Qualikems Fine Chem Pvt. Ltd., Vadodara, India. Glacial acetic acid was procured from Merck, Mumbai, India. Analytical grade other reagents and chemical were employed in the experiments. Purified water was employed throughout the experimental procedure.

\section{Preformulation and Physicochemical Characterization of Mesalamine}

Before the development of any dosage form, it is necessary to know several physiochemical properties (preformulation studies) of the drug. This information helps the formulator to develop an elegant, stable, effective and safe dosage form. In addition, preformulation study also aids in the authentication of the drug. Several preformulation factors were evaluated for mesalamine before going for formulation of beads.

\section{Melting Point Determination}

It is one of the easiest ways to determine the purity of the drug. The melting point of the mesalamine was determined by capillary tube method. The open end 
of the melting point capillary was filled with the small amount of the drug and tapped several times so that drug reaches to the bottom of closed end. The capillary was placed in digital melting point apparatus. The apparatus was heated initially at a rate of $10^{\circ} \mathrm{C} / \mathrm{min}$ and then $1{ }^{\circ} \mathrm{C} / \mathrm{min}$ near to the expected melting point. The temperature at which melting of the drug take place was noted down.

\section{$U V$-wavelength $\left(\lambda_{\max }\right)$}

Accurately weighed mesalamine drug $(10 \mathrm{mg})$ was dissolved in $10 \mathrm{ml}$ volumetric flask containing methanol. Then volume was adjusted upto the level with sufficient quantity of methanol. This gave the concentration of $1000 \mu \mathrm{g} / \mathrm{ml}$. It was diluted to $100 \mu \mathrm{g} / \mathrm{ml}$. Further, different aliquots were prepared with the stock solution. These aliquots were analyzed spectrophometrically (Shimadzu, UV-1800, Japan).

\section{Preparation of Standard Curve}

Mesalamine (10 mg) was accurately weighed and dissolved in $100 \mathrm{ml}$ volumetric flask containing hydrochloric acid buffer (0.1N HCL, pH 1.2) and simulated gastric fluid (SGF, pH 6.8, pH 7.4) individually. To get a $100 \mu \mathrm{g} / \mathrm{ml}$ concentration in the buffer medium of $\mathrm{pH} 1.2, \mathrm{pH} 6.8$ and $\mathrm{pH} 7.4$ volume was maintained upto level separately. This stock solution was used to prepare further standard solution of the drug. From the standard stock solution, different aliquots $0.2,0.4,0.6,0.8,1.0,1.2,1.4,1.6,1.8$ and $2.0 \mathrm{ml}$ were taken from different buffer $\mathrm{pH}$ and volume made up to $10 \mathrm{ml}$ with each buffer $\mathrm{pH} 1.2, \mathrm{pH} 6.8$ and $\mathrm{pH} 7.4$ individually in volumetric flask to produce 2, 4, 6, 8, 10, 12, 14, 1618 and $20 \mu \mathrm{g} / \mathrm{ml}$ respectively. The solutions were filtered (Whatman filter paper \# 41) and absorbance was measured at $220 \mathrm{~nm}$ using a doublebeam UV-spectrophotometer (Shimadzu, UV-1800, Japan).

\section{Solubility Studies}

Solubility is an important preformulation study which helps the formulator to choose the best solvent for the drug and enables the formulation of drug in an appropriate dosage form. The solubility of the drug was determined by taking excess amount of drug in $1 \mathrm{ml}$ of a specific solvent in a glass vial and subjected to shaking for $12 \mathrm{~h}$. Further the vial was kept aside till the undissolved drug settle down. The supernatant was collected and drug content was determined spectrophotometrically $\left(\lambda_{\max } 220 \mathrm{~nm}\right)$.

\section{FT-IR Analysis}

FTIR spectrum investigation was carried out to confirm the compatibility of pure drug mesalamine with different excipients which was used for preparation of optimized mesalamine loaded mucoadhesive beads. The $\mathrm{KBr}$ discs of pure mesalamine, polymers (sodium alginate, carbopol-940, guar gum) and powdered mesalamine brads were prepared and scanned in an FTIR spectrophotometer (Shimadzu IR Affinity, Japan) at 4000$500 \mathrm{~cm}^{-1}$ wave number.

\section{Differential Scanning Calorimeter (DSC) Studies}

The thermal behavior of mesalamine was studied in Perkin Elmer, Pyris Diamond DSC, USA at a heating rate of $10^{\circ} \mathrm{C} / \mathrm{min}$. Accurately weight sample (5mg) was sealed in an aluminum pan and measurement was performed at a heating range of $50-370^{\circ} \mathrm{C}$. Before analysis, equipment was standardized with zinc and indium.

\section{Preparation and Coating of Mucoadhesive Beads}

Mesalamine loaded mucoadhesive beads were prepared by ionic gelation method as reported by Deshmukh et al. (2020) with slight modification. Briefly, sodium alginate (SA 3\%, w/v) was dissolved in distilled water (DW). Carbopol 940 (CP; 250, 500, 750, 350 and $150 \mathrm{mg}$ ) and guar gum (GG; 500, 500, 250, 150 and $100 \mathrm{mg}$ ) were dissolved in $25 \mathrm{ml} \mathrm{DW}$ separately and kept in room temperature for $24 \mathrm{~h}$ to swell completely. The CP and GG dispersion were mixed together at equal ratio (1:2, $1: 1,3: 1,2.33: 1$ and 1.5:1 w/w) under magnetic stirrer (Remi, India) at specific stirring rate for $1 \mathrm{~h}$ in order to find homogeneous mass of both the gum. Mesalamine (250 mg) was then added in to gum slurry. This gum slurry was added in SA solution and mixed properly at an appropriate stirring rate by using magnetic stirrer as shown in Table 1. Cross linking solution was prepared by dissolving calcium chloride $\left(\mathrm{CaCl}_{2}, 5 \% \mathrm{w} / \mathrm{v}\right)$ in $\mathrm{DW}$ containing glacial acetic acid (GAA, 10\% v/v).

To ensure the drug and gum mixture was free from air bubbles before its use. Then the drug and gum mixture were added drop-wise to the cross-linking solutionthrough a disposable syringe needle ( $24 \mathrm{G}$ size).

\begin{tabular}{|c|c|c|c|}
\hline \multicolumn{4}{|c|}{ Table 1: Formulation of mesalamine loaded } \\
mucoadhesive beads. \\
\hline $\begin{array}{c}\text { Formulation } \\
\text { code }\end{array}$ & $\begin{array}{c}\text { Drug: } \\
\text { Polymer } \\
\text { ratio (mg, } \\
\text { w/w) }\end{array}$ & $\begin{array}{c}\text { Polymer } \\
\text { ratio (CP: } \\
\text { GG) (mg, } \\
\text { w/w) }\end{array}$ & $\begin{array}{c}\text { Stirring rate } \\
\text { (rpm) }\end{array}$ \\
\hline MA-F1 & $1: 1(250: 250)$ & $\begin{array}{c}1.5: 1 \\
(150: 100)\end{array}$ & 500 \\
\hline MA-F2 & $1: 2(250: 500)$ & $\begin{array}{c}2.33: 1 \\
(350: 150)\end{array}$ & 500 \\
\hline MA-F3 & $1: 3(250: 750)$ & $1: 2(250: 500)$ & 500 \\
\hline MA-F4 & $\begin{array}{c}1: 4 \\
(250: 1000)\end{array}$ & $1: 1(500: 500)$ & 500 \\
\hline MA-F5 & $\begin{array}{c}1: 4 \\
(250: 1000)\end{array}$ & $3: 1(750: 250)$ & 500 \\
\hline
\end{tabular}


The drug loaded micro beads were formed immediately and kept a side for $30 \mathrm{~min}$ to complete the reaction. The mesalamine loaded beads were collected by filtration and washed with DW 4-5 times to remove the $\mathrm{CaCl}_{2}$ residues from the beads. Beads were dried under hot air oven at medium temperature 55 for $3 \mathrm{~h}$. Spherical dried mesalamine - mucoadhesive beads were packed into air tight vials and stored in desiccators for further studies. Similarly, the placebo (without drug) mucoadhesive beads were also prepared.

The prepared mucoadhesive beads were coated with an enteric coating solution (Eudragit S 100) for protection of beads to release its content in upper part of GIT as described by Deshmukh et al..$^{25}$ Briefly, Eudragit S 100, enteric coating polymer $(2.98 \mathrm{~g})$ was dissolve in $25 \mathrm{ml}$ mixture of isopropyl alcohol and acetone (4:3). PEG-400 $(3 \% \mathrm{w} / \mathrm{v})$, a plasticizer was also added to the solution. The microparticles were coated by this solution by dip coating method till the weight increase by $10 \%$ and further dried in hot air oven at $40^{\circ} \mathrm{C}$ for $4 \mathrm{~h}$.

\section{Evaluation of Mucoadhesive Beads of Mesalamine Particle Size and Zeta Potential Analysis}

Zetasizer (Nano ZS90, Malvern instruments Ltd., UK) was utilized for size and zeta potential analysis. The mesalamine loaded beads were suspended in distilled water and analyzed in Zetasizer for suitable particle size and zeta potential.

\section{Percentage Drug Entrapment Efficiency (\%EE), Drug Loading $(\% D L)$ and Yield}

UV-vis spectrophotometer (Shimadzu, UV-1800, Japan) was used for this analysis. $\% \mathrm{EE}, \% \mathrm{DL}$ and $\%$ yield of the mesalamine loaded mucoadhesive beads (MA-F15) were determined as describe by Anande et al. 2008. About $100 \mathrm{mg}$ of the beads were crushed and made to powder in a glass mortar. Further, to release the drug content the powder was extracted out in SGF $(100 \mathrm{ml})$ by using bath sonicator. Thereafter, the suspension was filtered through membrane filters $(0.45 \mu \mathrm{m})$ and analyzed spectrophotometrically at $220 \mathrm{~nm} .{ }^{26}$ Each determination was made in triplicate. Analysis was performed in triplicate $(n=3)$. The $\% \mathrm{EE}, \% \mathrm{DL}$ and yield were calculated according to the following equations:

$$
\begin{gathered}
\% \mathrm{EE}=\frac{\text { Calculated drug content }}{\text { Theoretical drug content }} \times 100 \\
\text { Total amount of drug in beads }- \\
\% \mathrm{DL}=\frac{\text { Amount of free drug }}{\text { Total weight of beads }} \times 100
\end{gathered}
$$

$$
\begin{gathered}
\% \text { Yield }= \\
\begin{array}{c}
\text { Total weight of drug, polymer } \\
\text { and other non }- \text { volatile solids }
\end{array}
\end{gathered}
$$

\section{Swelling Index}

The swelling extent of beads (MA-F1-5) was carried out in phosphate buffer of $\mathrm{pH} 6.8$ and 7.4. An exactly weighed $100 \mathrm{mg}$ of beads were transferred to the basket of USP type I dissolution test apparatus and allowed to swell for $12 \mathrm{~h}$ at $37 \pm 0.1^{\circ} \mathrm{C}$. Thereafter, the fluid adhering with beads were wiped out by using filter paper and swelled beads were taken out for weight measurement. Swelling index is calculated according to the following formula. ${ }^{27}$

$$
\% \text { Degree of swelling }(\alpha)=\frac{\omega_{\mathrm{s}}-\omega_{0}}{\omega_{0}}
$$

Where, $\omega_{0}=$ weight of un swollen beads and $\omega_{\mathrm{s}}=$ weight of swollen beads.

\section{Mucoadhesion Testing by In vitro Wash-Off Method}

The mucoadhesive properties of mesalamine-loaded beads (MA-F1-5) were evaluated by the in vitro washoff technique as describe by Malik et al. ${ }^{28}$ Briefly, goat intestinal mucosa was collected from local slaughter house and a size of $1 \times 1 \mathrm{~cm}$ was excised and fixed into the glass slide with the help of thread. The beads (100 in count) were then sprinkled over the mucosa and then to an arm of tablet disintegration test apparatus. The samples were subjected to up and down movement in beaker containing $900 \mathrm{ml}$ of phosphate buffer $(\mathrm{pH}$ 7.4) maintained at $37 \pm 0.5^{\circ} \mathrm{C}$. The beads were observed regularly for adherence with intestinal mucosa and total beads adhered were recorded for a period of $12 \mathrm{~h}$. The percentage mucoadhesive efficiency of the beads was recorded according to the formula:

$$
\% \text { Mucoadhesion }=\frac{\text { Number of adhered of beads }}{\text { Total number of applied of beads }} \times 100
$$

\section{Surface Electron Microscopy (SEM) Study}

The architecture of MA-F4 beads (shape and surface) was studied by using SEM (Jeol, JSM-1600, Tokyo, Japan). Prior to imaging, the beads were sprinkled over sided adhesive carbon tape which was stuck to aluminum stub. Further it was subjected to gold coating using gold sputter under inert atmosphere in a highvacuum evaporator. These specimens were analyzed and their photomicrographs were recorded at different magnifications. 


\section{In vitro Drug Release Study}

The in vitro drug release study from novel mesalamine formulations (MA-F1-5) and marketed tablet formulation of mesalamine (Pentasa $250 \mathrm{mg}$ ) was performed according to the procedure reported by Anande $e t$ al. Multiple dissolution rate test (six paddles) apparatus was used for performing the release profile of the formulations. A simulated GI fluid (SGF) was used as the dissolution medium. Mesalamine beads (100 $\mathrm{mg}$ ) were weighed accurately and put into the beaker containing $900 \mathrm{ml}$ SGF. The dissolution content was rotated at $100 \mathrm{rpm}$ at $37 \pm 0.5^{\circ} \mathrm{C}$ and exact equilibrium (sink) condition was maintained during the study period. The different $\mathrm{pH}$ of SGF and GI transit conditions were obtained by changing the dissolution medium at different periods. In the Initial study period, pH 1.2 of SGF was maintained for $2 \mathrm{~h}$ with the help of $0.1 \mathrm{~N} \mathrm{HCl}$. Then accurately weighed quantity of potassium di-hydrogen phosphate $(1.7 \mathrm{~g})$ and di-sodium-hydrogen-phosphatedihydrate $(2.2 \mathrm{~g})$ were added in to the SGF medium, adjusting the $\mathrm{pH}$ to 6.8 using $1.0 \mathrm{M} \mathrm{NaOH}$ and the release rate study was continued for further $2 \mathrm{~h}$. After 4 $h$, the $\mathrm{pH}$ of SGF was changed to 7.4 with $0.1 \mathrm{~N} \mathrm{NaOH}$ for $24 \mathrm{~h}$. A $5 \mathrm{ml}$ sample was taken from the dissolution medium at regular time period and an equivalent volume of fresh SGF was replaced to preserve the sink conditions. Prior measurement, all the test solutions were filtered through the syringe filter $(0.45 \mu \mathrm{m}, \mathrm{NYL})$ and analyzed spectrophotometrically at $220 \mathrm{~nm}$.

\section{Stability Study}

MA-F4 was tested for stability study according to the guidelines of ICH. It was tested at $25 \pm 2 / 60 \pm 5 \%$ $\mathrm{RH}, 30 \pm 2 / 65 \pm 5 \% \mathrm{RH}$ and $40 \pm 2 / 75 \pm 5 \% \mathrm{RH}$ for 180 days. The changes were observed periodically for 45, 90 and 180 days and shelf life were calculated for storage condition at different temperatures.

\section{Statistical Analysis}

A one-way analysis of variance (ANOVA) was performed for analysis of the experimental data. Graph Pad Prism software-5, San Diego, CA, USA was used for statistical analysis of the data. All the data were determined by the mean \pm standard deviation (SD) and mean variations were considered to be significant at $P<0.05$.

\section{RESULTS}

\section{Physicochemical Characterization of Mesalamine Melting Point}

M. P. of mesalamine was found to be $282^{\circ} \mathrm{C}$. But in this temperature drug started to decompose.
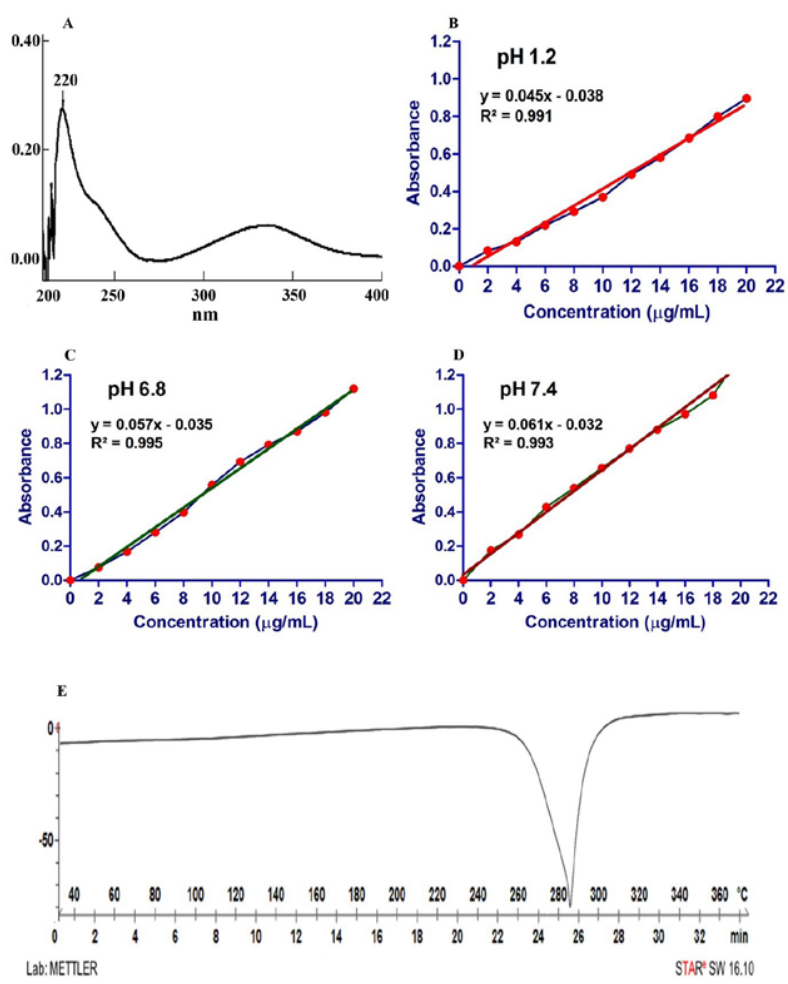

Figure 1: Preformulation of mesalamine. (A) UV-Visible spectra of mesalamine (B) Calibration curve of mesalamine in SGF $(\mathrm{pH} 1.2)$ at $220 \mathrm{~nm}$ (C) Calibration curve of mesalamine in SGF (pH 6.8) at $220 \mathrm{~nm}$ (D) Calibration curve of mesalamine in SGF (pH 7.4) at $220 \mathrm{~nm}$ (E) DSC of the mesalamine.

\section{Determination of Absorbance Maxima $\left(\lambda_{\max }\right)$}

The mesalamine dissolved in methanol and diluted to give solution of $10 \mu \mathrm{g} / \mathrm{ml}$ which was spectrophotometrically analyzed at UV range. Solution gives peak at $220 \mathrm{~nm}$ as shown in Figure 1A. Thus, this wavelength was selected as $\lambda_{\text {max }}$ for the further study.

\section{Preparation of Standard Curve}

The stock solution of mesalamine was diluted with either hydrochloric acid buffer $(0.1 \mathrm{~N} \mathrm{HCl}, \mathrm{pH} 1.2)$, SGF $\mathrm{pH} 6.8, \mathrm{pH} 7.4$ to get a concentration range of 2 to 20 $\mu \mathrm{g} / \mathrm{ml}$ of mesalamine. The absorbance was measured at $220 \mathrm{~nm}$ and calibration curve of mesalamine was plotted between absorbance and concentration for the 3 different buffers solutions was shown in Figure 1 B-D. The data were subjected to linear regression and the correlation coefficient was found to be 0.9903 for $\mathrm{pH} \mathrm{1.2,} 0.995$ for $\mathrm{pH} 6.8$ and 0.9943 for $\mathrm{pH}$ 7.4. Thus, the value of correlation coefficient indicates that the concentration range of 2 to $20 \mu \mathrm{g} / \mathrm{ml}$ of mesalamine obeys beer's lambert law.

\section{Differential Scanning Calorimeter Studies}

The DSC spectrum of mesalamine shows a sharp endothermic peak at $282^{\circ} \mathrm{C}$ (Figure 1E). This was in accordance to the obtained melting point of the 
Table 2: Solubility study of mesalamine in different solvents.

\begin{tabular}{|c|c|}
\hline Solvents & Solubility \\
\hline Distilled water & Insoluble \\
\hline 0.1N HCl solution. & Insoluble \\
\hline Phosphate buffer of $\mathrm{pH} 6.8$ & Insoluble \\
\hline Phosphate buffer of $\mathrm{pH} \mathrm{7.4}$ & Insoluble \\
\hline Methanol & Soluble \\
\hline DMSO & Freely soluble \\
\hline Acetone & Freely soluble \\
\hline
\end{tabular}

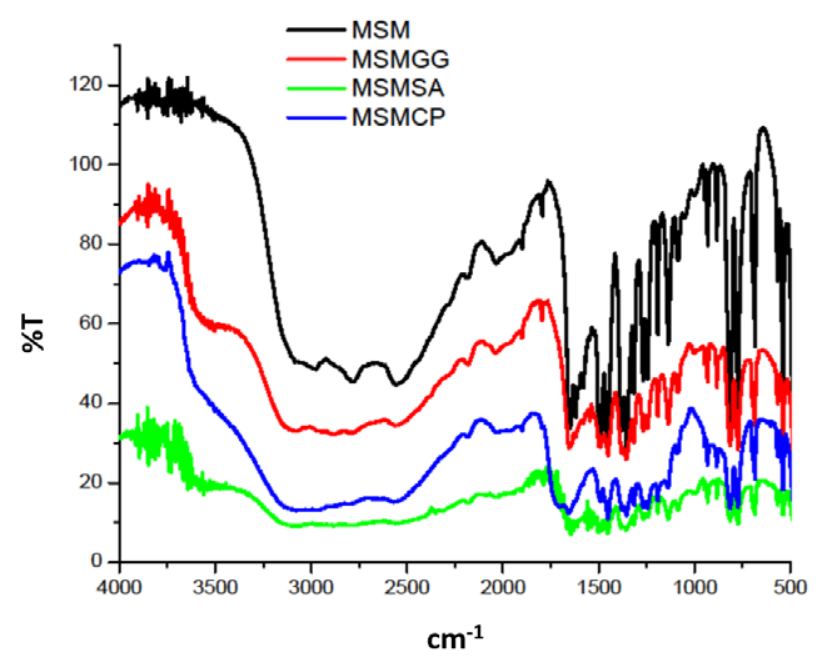

Figure 2: Overlayed FTIR spectrum of mesalamine with the ingredients of formulation. MSM: mesalamine, MSMGG: mesalamine and guargum, MSMSA: mesalamine and sodium alginate, MSMCP: mesalamine and carbopol.

mesalamine. Thus, this study proves the structural stability of the mesalamine.

\section{Solubility Studies}

The solubility mesalamine in different solution was given in Table 2.

\section{FTIR Analysis}

The drug-excipient interaction FTIR spectrum has shown in Figure 2. Drug sample FTIR spectrum data was interpreted and matched with standard FTIR spectra of mesalamine which confirms the authenticity of the sample drug by identifying peaks as similar as reference drug. The result shows that the mesalamine shows band at $1649.14 \mathrm{~cm}^{-1}$ ( $\mathrm{C}=\mathrm{O}$ stretching), $1489.05 \mathrm{~cm}^{-1}(\mathrm{C}=\mathrm{C})$, 1452.4 $\left(\mathrm{CH}_{2}\right.$ bending), $1355.96 \mathrm{~cm}^{-1}(\mathrm{C}-\mathrm{N})$. In addition, FTIR spectrum analysis of drug and components of formulation was also performed. There were very slightly shift of drug in to polymer matrix. Thus, the spectrum proves that the drug mesalamine was quite compatible with its polymer used in development of mucoadhesive beads.

The results of preformulation studies of mesalamine were in accordance to standard and therefore it can be concluded that the drug was authentic. Moreover, the drug was compatible with the other ingredients used for the formulation of the microparticles.

\section{Development of Mesalamine Mucoadhesive Beads}

Mesalamine loaded different mucoadhesive beads were developed by an appropriate ionic-gelation technique as describe by Deshmukh et al. with slight modifications. ${ }^{25}$ Beads were prepared by using different drug: polymer ratios $(\mathrm{w} / \mathrm{w})$. Time of cross linking between $5 \% \mathrm{CaCl}_{2}$ and sodium alginate-based CP/GG slurry is a key point for formation of spherical micro beads at specific stirring rates under magnetic stirrer. The optimized stirring rate was found to be $500 \mathrm{rpm}$ for fabricating mesalamine loaded appropriate mucoadhesive beads. The beads were then coated with Eudragit S100 until weight of the beads increases by $10 \%-12 \%$ by its initial weight.

\section{Evaluation of Mucoadhesive Beads of Mesalamine Particle Size and Zeta Potential Analysis}

The dissolution and absorption of a drug from formulation is influence by its particle size. Zeta potential value indicates the stability of the formulation. Therefore, the particle size and zeta potential of the mesalamine loaded mucoadhesive beads of all the formulation (MA-F1 to MA-F5) were analyzed. The size of the beads was found to be in size range of 445.6 $\pm 67.1 \mu \mathrm{m}$ to $842.1 \pm 76.9 \mu \mathrm{m}$ and zeta potential was found to be in range of $-24.14 \pm 0.21 \mathrm{mV}$ to $-31.6 \pm$ $0.26 \mathrm{mV}$. As the concentration of polymer increases the mean particle size also increases Table 3 . It is well known that as the particle size decrease the surface area increases which may facilitate the drug absorption from the formulations. Hence, it is desirable that the optimized formulation should have a less particle size for drug to release from the formulation. A zeta potential result indicates that the formed beads are negatively charged and as a result they will have a good stability. The size distribution and zeta potential of MA-F4 was given in Figure 3.

Surface structure and morphology of the optimized formulation, MA-F4 has been visualized through SEM (Figure 4). Photomicrograph of the uncoated particle was rough in shape while the beads coated with Eudragit S-100 were seen as a smooth and spherical in shape. Thus, the coating helps in smoothing the surface of the beads. 


\begin{tabular}{|c|c|c|c|c|c|}
\hline \multicolumn{6}{|c|}{ Table 3: Characterization of mesalamine loaded different mucoadhesive beads. } \\
\hline Formulation code & Particle size $(\boldsymbol{\mu m})$ & Zeta potential $(\mathbf{m V})$ & \%EE & \%Yield & \%DL \\
\hline MA-F1 & $842.1 \pm 76.9$ & $-25.93 \pm 0.30$ & $72.28 \pm 6.54$ & $74.51 \pm 6.45$ & $23.4 \pm 0.6$ \\
\hline MA-F2 & $755.4 \pm 60.2$ & $-24.14 \pm 0.21$ & $91.33 \pm 3.63$ & $83.34 \pm 5.85$ & $28.5 \pm 0.9$ \\
\hline MA-F3 & $457.3 \pm 56.7$ & $-24.92 \pm 0.28$ & $82.52 \pm 2.14$ & $82.58 \pm 4.26$ & $20.8 \pm 0.7$ \\
\hline MA-F4 & $445.6 \pm 67.1$ & $-28.01 \pm 0.16$ & $94.98 \pm 3.22$ & $96.27 \pm 3.22$ & $30.4 \pm 0.9$ \\
\hline MA-F5 & $667.9 \pm 86.4$ & $-31.6 \pm 0.26$ & $88.43 \pm 3.11$ & $87.83 \pm 3.18$ & $30.1 \pm 0.8$ \\
\hline
\end{tabular}
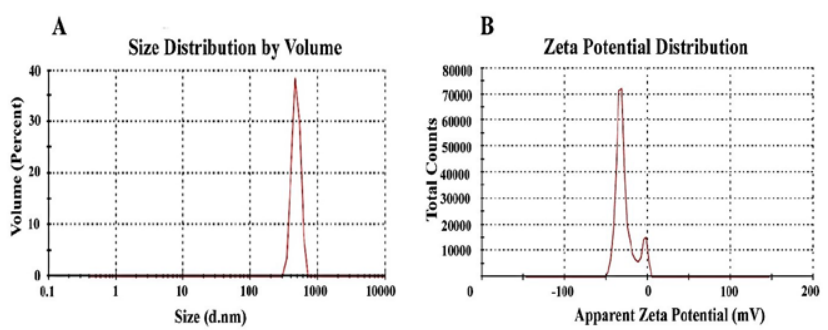

Figure 3: Particle size distribution (A) and zeta potential (B) of the optimized formulation MA-F4.
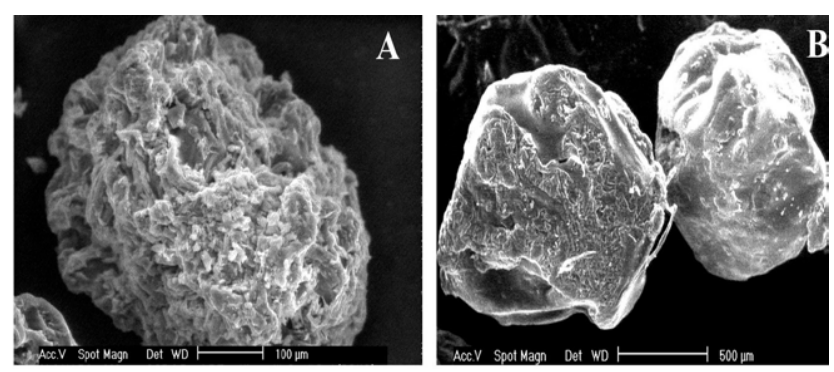

Figure 4: SEM photomicrographs of mesalamine loaded uncoated mucoadhesive beads $(A)$ and mesalamine loaded Eudragit S-100 coated mucoadhesive beads (B).

\section{Drug entrapment Efficiency, Drug Loading and Percent Yield}

$\%$ EE, \% drug loading capacity and \% yield of the different formulations, MA-F1-5 were calculated and results have been represented in Table 3. MA-F4 showed highest value than that of others. The entrapment efficiency was found to be $94 \pm 3 \%$, drug loading was $94 \pm 3 \%$ and $\%$ yield was $96 \pm 3$. The formulation MA-F4 contain 1:1 w/w polymer ratio (CP: GG). This ratio was optimal for development of beads at constant $500 \mathrm{rpm}$. Therefore, the formulation MA-F4 was chosen as an optimized formulation.

\section{Degree of Swelling or Swelling Index (SI)}

Swelling efficiency of MA-F1-F5 were evaluated in simulated intestinal fluid at $\mathrm{pH} 6.8$ and $\mathrm{pH} 7.4$ and results were shown in Figure $5(\mathrm{~A})$. Swelling indexes $(\theta)$ were found to be in the range of $0.87 \pm 0.09$ to $1.25 \pm$ 0.05 at $\mathrm{pH} 6.8$ and in range of $0.86 \pm 0.05$ to $1.28 \pm 0.04$ at $\mathrm{pH}$ 7.4. The best SI $(\theta)(1.25 \pm 0.05$ at $\mathrm{pH} 6.8$ and $1.28 \pm 0.04$ at $\mathrm{pH} 7.4)$ value was achieved with MA-F4
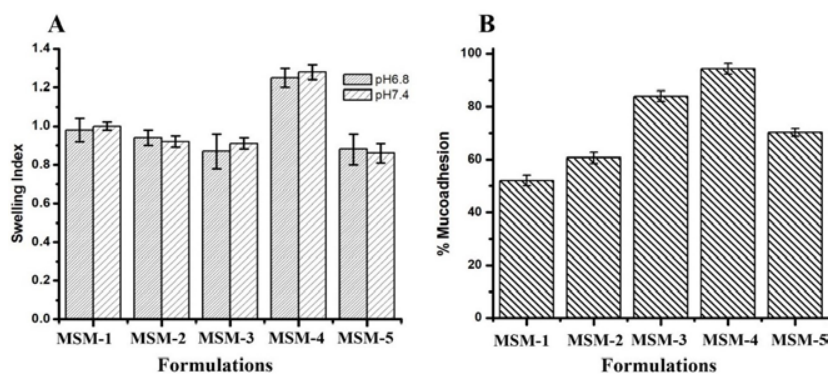

Figure 5: Swelling index (A) and mucoadhesion of mesalamine loaded mucoadhesive beads (B). Values were represented as mean $\pm \operatorname{SD}(n=3)$.

for an extended period ( $24 \mathrm{~h}$ ) as compared to other formulations, which is suitable for retaining in colon.

\section{Mucoadhesion}

Mucoadhesion of various formulations, MA-F1-5 were performed in goat intestinal mucosa to check out the adhesion efficiency of beads to the intestinal mucosa for prolonged release of drug. Results were shown in Figure 5(B). Mucoadhesion were found to be from $60.67 \%$ $\pm 2.1 \%$ to $94.33 \% \pm 2.1 \%$ for different formulations. The maximum mucoadhesion $(94.33 \% \pm 2.1 \%)(P<$ 0.001) was achieved with MA-F4 which was statically significantly as compared to other formulations.

\section{In vitro Drug Release}

Mesalamine release profile of MA-F1-5 and marketed tablet Pentasa was studied in $\mathrm{pH}$ progression medium (SGF) at $37 \pm 0.5^{\circ} \mathrm{C}$. The maximum mesalamine content was found to be $95.07 \pm 3.86 \%$ significantly from MA-F4 than that of other formulations including Pentasa as shown in Figure 6A. Cumulative \% release profile from MA-F1-5 exhibited the desired rate as there was no quantifiable drug released at $\mathrm{pH} 1.2$ for $2 \mathrm{~h}$ while at $\mathrm{pH} 6.8$ the drug release was quite insignificant for period of $4 \mathrm{~h}$. As represented in Figure 6B, $17.18 \pm$ $2.83 \%$ mesalamine was released from Pentasa tablet for $2 \mathrm{~h}$ at $\mathrm{pH} 1.2$ but in case of MA-F4 very less amount $(>1.60 \pm 0.53 \%)$ of mesalamine was released for $2 \mathrm{~h}$ at $\mathrm{pH}$ 1.2. At $\mathrm{pH} 6.8,26.01 \pm 8.99 \%$ drug content was released from Pentasa tablet, while mesalamine was released $3.83 \pm 0.43 \%$ from MA-F4 after $4 \mathrm{~h}$. The drug released from beads at acidic $\mathrm{pH} 1.2$ which may be due to 
the permeation process through membrane but released in $\mathrm{pH} 7.4$ could follow both matrix erosion and diffusion pattern of drug release. Mesalamine $97.16 \pm 3.92 \%$ was released from the Pentasa tablet for period of $12 \mathrm{~h}$ at $\mathrm{pH} 7.4$ because of uncoated tablet. Formulation MA-F4 exhibited remarkable sustained release profile (95.07 $\pm 3.85 \%$ ) for $24 \mathrm{~h}$ at $\mathrm{pH} 7.4$ as compared to Pentasa tablet, which may be due to mucoadhesive property of the sodium alginate based carbopol 940 and guar gum coated beads.

\section{Drug Release Kinetics}

Different kinetic treatments were applied to interpret the release of mesalamine from different matrices which has been represented in Table 4. The best formulation, MA-F4 followed Korsmeyer's Peppas model having $r^{2}=0.9236$ and diffusion exponent value $n=1.6442$. So
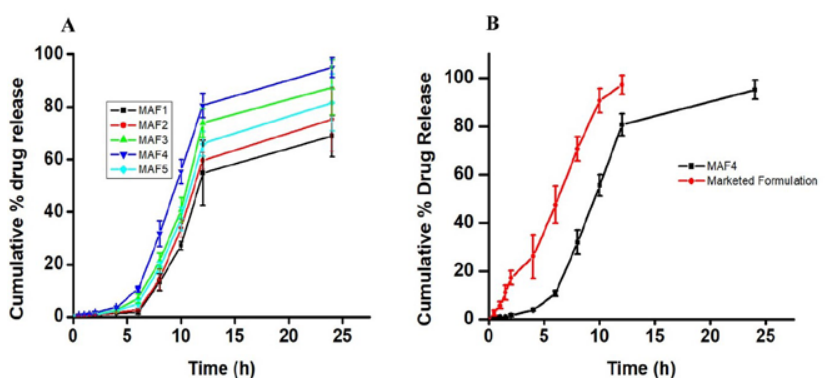

Figure 6: In vitro drug release: (A) Release profile of mesalamine loaded mucoadhesive beads (MA-F1-5) at pH 1.2, 6.8 and 7.4. (B) Comparative release profile of optimized beads, MA-F4 and Pentasa tablet at $\mathrm{pH} 1.2,6.8$ and 7.4. The values were mean \pm SD $(n=3)$.
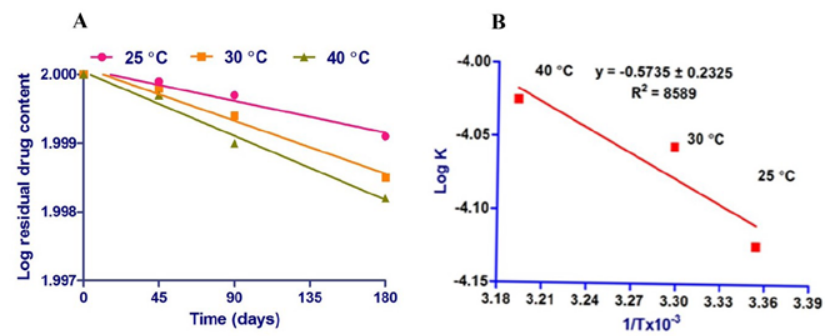

Figure 7: First order (A) and Arrhenius plot (B) of MA-F4 for degradation at different storage temperatures $\left(25^{\circ} \mathrm{C}, 30^{\circ} \mathrm{C}\right.$ and $40^{\circ} \mathrm{C}$ ). the drug release mechanism was super Case-II transport and non-Fickian pattern. ${ }^{29}$

\section{Stability Studies}

The MA-F4 formulation was kept inside amber color bottled and sealed with aluminum foil and evaluated for stability. At a regular interval of $0,45,90$ and 180 days the formulation was analyzed for its size, zeta potential and residual drug content. Result suggests that the prepared formulation was stable at room temperature. With the increase of temperature there was increase in rate of degradation [first order, Figure 7(A)], decrease in the size and zeta potential. The shelf-life of MA-F4 was predicted from Arrhenius plot and was found to be $3.84,3.29$ and 3.06 years at $25^{\circ} \mathrm{C}, 30^{\circ} \mathrm{C}$ and $40^{\circ} \mathrm{C}$ respectively [Figure $7(\mathrm{~B})$ ]. The data of particle size and residual drug content at different temperature were also subjected to t-test with $95 \%$ significant level and $P<0.05$.

\section{DISCUSSION}

IBD is concerned with GIT problems, particularly small and large intestine consisting of (UC) and (CD). It may be either progressive or chronic with unknown etiology effecting millions of people worldwide and affecting the quality of life of people. Anti-inflammatory drugs especially 5ASA are the first choice of treatment for management of IBD. The oral is the most appropriate and choice of drug delivery with high patience compliances. But the conventional drug delivery has high dose size and dose frequency resulting in increased side effect. Therefore, it is desired that the delivery system should work locally at the colon site or can be systemically absorbed from the colon and simultaneously protecting the drug from exposure to gastric enzymes, altered $\mathrm{pH}$ conditions and variations in gastric voiding. Some of the most common approaches used to deliver drug to the colon includes use of polymers releasing drug dependent on $\mathrm{pH}$, time-release coatings, prodrugs and biodegradable polymers. ${ }^{30-32}$ In last few years the utilization of polymeric microparticles and beads for colon targeting has become a promising approach. ${ }^{33} \mathrm{~A}$

\begin{tabular}{|c|c|c|c|c|c|c|c|c|}
\hline \multicolumn{8}{|c|}{ Table 4: Kinetic treatment of prepared mesalamine beads formulations. } \\
\hline \multirow{2}{*}{ Formulations } & \multicolumn{2}{|c|}{ Zero order } & \multicolumn{2}{c|}{ First order } & \multicolumn{2}{c|}{ Higuchi square root } & \multicolumn{2}{c|}{ Korsmeyer plot } \\
\cline { 2 - 9 } & $\boldsymbol{r}^{\mathbf{2}}$ & $\boldsymbol{k}$ & $\boldsymbol{r}^{\mathbf{2}}$ & $\boldsymbol{k}$ & $\boldsymbol{r}^{2}$ & $\boldsymbol{k}$ & $\boldsymbol{r}^{\mathbf{2}}$ & $\boldsymbol{n}$ \\
\hline MA-F1 & 0.8747 & 3.2295 & 0.8846 & 0.0226 & 0.7515 & 15.021 & 0.8384 & 1.583 \\
\hline MA-F2 & 0.879 & 3.5516 & 0.8962 & 0.0269 & 0.7629 & 16.603 & 0.7261 & 1.6232 \\
\hline MA-F3 & 0.8778 & 4.1899 & 0.8944 & 0.0396 & 0.7823 & 19.859 & 0.7011 & 1.4403 \\
\hline MA-F4 & $\mathbf{0 . 8 8 9 1}$ & $\mathbf{4 . 6 5 4 3}$ & $\mathbf{0 . 9 2 4 9}$ & $\mathbf{0 . 0 5 6 2}$ & $\mathbf{0 . 8 1 8 5}$ & $\mathbf{2 2 . 4 9 9}$ & $\mathbf{0 . 9 2 3 6}$ & $\mathbf{1 . 6 6 4 2}$ \\
\hline MA-F5 & 0.8863 & 3.8803 & 0.9033 & 0.0325 & 0.7818 & 18.287 & 0.72 & 2.3586 \\
\hline
\end{tabular}


wide variety of polymers (natural and synthetic) have gained prominence in pharmaceutical research for developing technology to administer drug directly to the colon. Hence, the present study was aimed to formulate a microparticles carrying mesalamine in the blend of polymer matrix for an effective delivery to colon against IBD. The choice of treatment for IBD involves the use of colon specific anti-inflammatory drug like mesalamine. Yet the actual mechanism of action of mesalamine is doubtful but it was believed that it diminishes inflammation by blocking cyclooxygenase and inhibiting prostaglandin production in the colon.

The anti-inflammatory drug mesalamine was subjected to preformulation studies for its authentication and it was observed that the procured drug has a Melting point and $\lambda_{\text {max }}$ was found to be $282^{\circ} \mathrm{C}$ and $220 \mathrm{~nm}$ respectively. DSC study (Figure 1E) gives a sharp endothermic peak at $282^{\circ} \mathrm{C}$ was similar to MP of drug. In addition, the FTIR spectrum of drug was compared with standard a spectrum which was almost similar. Thus, the various preformulation studies confirm the purity and stability of the mesalamine.

The mucoadhesive beads were prepared by ion gelation technique using $5 \%$ acidified $\mathrm{CaCl}_{2}$ solution. Total 5 different batches of formulation (MA-F1 to MA-F5) were prepared and subjected for various characterization and evaluation.

The particle size and zeta potential of the beads was found to be in size range of $445.6 \pm 67.1 \mu \mathrm{m}$ to 842.1 $\pm 76.9 \mu \mathrm{m}$ and $-24.14 \pm 0.21 \mathrm{mV}$ to $-31.6 \pm 0.26$ $\mathrm{mV}$ respectively. Beads size is varied with polymeric concentration which has shown in Table 3. The increase polymeric concentration results in increased viscosity of the dispersion which may results in particle size. But it is always desired to have beads with less particle size as they have high surface area. Among all the batches MA-F4 had a least particle size of $445.6 \pm 67.1 \mu \mathrm{m}$

It is well known that as the particle size decrease the surface area increases which may facilitate the drug absorption from the formulations. Hence, it is desirable that the optimized formulation should have a less particle size for drug to release from the formulation. A zeta potential result indicates that the formed beads are negatively charged and as a result they will have a good stability. Moreover, the charge on the beads confers a good mucoadhesion with the mucosa of the colon via electrostatic interactions which may prolonged the colonic or intestinal residence time for the formulation. The size distribution and zeta potential of MA-F4 was given in Figure 3. The in vitro release of mesalamine from different formulations (MA-F1-5) follows the order of MA-F4 > MA-F3 > MA-F5 > MA-F2 > MA-F1.
Thus, the formulation MA-F4 exhibit highest drug releases of $95.07 \pm 3.86 \%$ in $24 \mathrm{~h}$ as compare to other formulations. The smaller particle size (increased surface area) and higher swelling index contributes to the maximum release of drug to the dissolution media. In contrast, formulation MA-F1 having highest particle size of $842.1 \pm 76.9 \mu \mathrm{m}$ exhibits lowest drug release profile as compare to other because of its low surface area, high crosslinking and low swelling efficiency. Therefore, based on results of different parameters the formulation MA-F4 was considered as best one among others. The stability study revealed that the formulation was most stable at $25 \pm 2{ }^{\circ} \mathrm{C}$ and the shelf life $\left(\mathrm{T}_{90}\right)$ was found to be 3.84 years. At elevated temperature there may be loss of moisture which may be responsible from change in texture, particle size and increase degradation rate. Thus, the mesalamine loaded polymeric shell microparticles coated with Eudragit S 100 can be a promising delivery system for colon targeting. Further, in vivo study is required to ensure the colon targeting for management of IBD.

\section{CONCLUSION}

IBD is an inflammatory disorder occurred in GIT especially at colon region. There were many conventional drug therapies available for the management and remission of the IBD. But the current conventional drugs delivery system has drawback of high dose size and frequency. Mucoadhesive microparticles played an important role in colon specific drug targeting. It can target the desired site with prolonged release profile for extended periods due to their adhesiveness property to the colonic mucosa. Hence, the objective of the current study was to develop and evaluate mesalamine loaded microparticles (beads) for colon targeting. The beads were developed by a blend of polymer (SA, GG and $\mathrm{CP})$ matrix system of the microparticles. The various preformulation studies help for the identification and establishing the purity of the drug. The beads had good mucoadhesive properties and release its content specifically in the colonic $\mathrm{pH}$ in sustained release manner. Hence, mucoadhesive beads would be pioneering in the treatment of IBD. Moreover, it can be good alternative to traditional delivery system which has drawback of high dose and more side effects.

\section{ACKNOWLEDGEMENT}

Authors are grateful to the Dr. Kamal Shah for FTIR studies and Dr. Prabal Pratap Singh for DSC studies. Authors are also thankful to IPR, GLA, University, Mathura, Uttar Pradesh for providing necessary facility to carryout work. 


\section{CONFLICT OF INTEREST}

Authors have no conflict of interest.

\section{ABBREVIATIONS}

ASA: Aminosalicilates; BCS: Biopharmaceutical Classification System; CD: Crohn's Disease; CP: Carbopol 940; DL: Drug Loading; DSC: Differential Scanning Calorimeter; DW: Distilled Water; EE: Entrapment Efficiency; GAA: Glacial Acetic Acid; GG: Guar Gum; IBD: Inflammatory Bowel Disease; PEG: Polyethylene Glycol; SA: Sodium Alginate; SEM: Scanning Electron Microscopy; SGF: Simulated Gastric Fluid; UC: Ulcerative Colitis.

\section{REFERENCES}

1. Podolsky DK. Inflammatory bowel disease. The New England Journal of Medicine. 2002;347:417-29.

2. Loftus EV. Clinical epidemiology of inflammatory bowel disease: Incidence, prevalence and environmental influences. Gastroenterology. 2004;126(6):1504-17.

3. Deshmukh R, Kumari S, Harwansh RK. Inflammatory Bowel Disease: A Snapshot of Current Knowledge. Research Journal of Pharmacy and Technology. 2020;13(2):956-62.

4. Hanić M, Trbojević-Akmačić I, Lauc G. Inflammatory bowel disease-glycomics perspective. Biochim Biophys Acta Gen Subj. 2019;1863(10):1595-601.

5. Koliani-Pace JL, Siegel CA. Prognosticating the Course of Inflammatory Bowel Disease. Gastrointestinal Endoscopy Clinics. 2019;29(3):395-404.

6. Agrawal M, Colombel JF. Treat-to-Target in Inflammatory Bowel Diseases, What Is the Target and How Do We Treat?. Gastrointestinal Endoscopy Clinics. 2019;29(3):421-36.

7. Sairenji T, Collins KL, Evans DV. An Update on Inflammatory Bowel Disease. Primary Care. 2017;44(4):673-92.

8. Im JP, Ye BD, Kim YS, Kim JS. Changing treatment paradigms for the management of inflammatory bowel disease. The Korean Journal of Internal Medicine. 2018;33(1):28-35.

9. Kedia S, Ahuja V. Epidemiology of Inflammatory Bowel Disease in India: The Great Shift East. Inflammatory Intestinal Diseases. 2017;2(2):102-15.

10. Hua S, Marks E, Schneider JJ, Keely S. Advances in oral nano-delivery systems for colon targeted drug delivery in inflammatory bowel disease: Selective targeting to diseased versus healthy tissue. Nanomedicine. 2015;11(5):1117-32.

11. Ham M, Moss AC. Mesalamine in the treatment and maintenance of remission of ulcerative colitis. Expert Review of Clinical Pharmacology. 2012;5(2):11323

12. Cross RK. Safety Considerations with the Use of Corticosteroids and Biologic Therapies in Mild-to-Moderate Ulcerative Colitis. Inflamm Bowel Dis. 2017;23(10):1689-701.

13. Hirten RP, lacucci M, Shah S, Ghosh S, Colombel JF. Combining Biologics in Inflammatory Bowel Disease and Other Immune Mediated Inflammatory Disorders. Clin Gastroenterol Hepatol. 2018;16(9):1374-84.
14. Biancone L, Onali S, Petruzziello C, Calabrese E, Pallone F. Cancer and immunomodulators in inflammatory bowel diseases. Inflamm Bowel Dis. 2015;21(3):674-98.

15. Bernstein $\mathrm{CN}$. Treatment of IBD: where we are and where we are going. Am J Gastroenterol. 2015;110(1):114-26.

16. Peppercorn MA. Sulfasalazine: Pharmacology, clinical use, toxicity and related new drug development. Annals of Internal Medicine. 1984;101(3):377-86.

17. Punchard NA, Greenfield SM, Thompson RP. Mechanism of action of 5-arninosalicylic acid. Mediators of Inflammation. 1992;1(3):151-65.

18. Tenjarla S. Dissolution of commercially available mesalamine formulations at various pH levels. Drugs in R and D. 2015;15(2):211-5.

19. Harwansh RK, Deshmukh R, Barkat MA, Rahman MA. Bioinspired Polymericbased Core-shell Smart Nano-systems. Pharmaceutical Nanotechnology. 2019;7(3):181-205.

20. Krishnaiah YS, Khan MA. Strategies of targeting oral drug delivery systems to the colon and their potential use for the treatment of colorectal cancer. Pharm Dev Technol. 2012;17(5):521-40.

21. Chakraborty S, Khandai M, Sharma A, Khanam N, Patra CN, Dinda SC, et al. Preparation, in vitro and in vivo evaluation of algino-pectinate bioadhesive microspheres: An investigation of the effects of polymers using multiple comparison analysis. Acta Pharm. 2010;60(3):255-66.

22. George A, Shah PA, Shrivastav PS. Guar gum: Versatile natural polymer for drug delivery applications. European Polymer Journal. 2019;112:722-35.

23. Agüero L, Zaldivar-Silva D, Peña L, Dias ML. Alginate microparticles as oral colon drug delivery device: A review. Carbohydrate Polymers. 2017;168:32-43.

24. Panzade P, Puranik PK. Carbopol Polymers: A Versatile Polymer for Pharmaceutical Applications. Research Journal of Pharmacy and Technology. 2010;3(3):672-5.

25. Deshmukh R, Harwansh RK, Paul SD, Shukla R. Controlled release of sulfasalazine loaded amidated pectin microparticles through Eudragit S 100 coated capsule for management of inflammatory bowel disease. Journal of Drug Delivery Science and Technology. 2020;55:101495.

26. Anande NM, Jain SK, Jain NK. Con-A conjugated mucoadhesive microspheres for the colonic delivery of diloxanide furoate. International Journal of Pharmaceutics. 2008;359(1-2):182-9.

27. Chaurasia M, Chourasia MK, Jain NK, Jain A, Soni V, Gupta Y, et al. Methotrexate bearing calcium pectinate microspheres: A platform to achieve colon-specific drug release. Curr Drug Deliv. 2008;5(3):215-9.

28. Malik RK, Malik P, Gulati N, Nagaich U. Fabrication and in vitro evaluation of mucoadhesive ondansetron hydrochloride beads for the management of emesis in chemotherapy. Int J Pharm Investig. 2013;3(1):42-6.

29. Dash S, Murthy P, Nath L, Chowdhury P. Kinetic Modeling on Drug Release from Controlled Drug Delivery Systems. Acta Poloniae Pharmaceutica 2010;67(3):217-23.

30. Kaur G, Jain S, Tiwary AK. Recent approaches for colon drug delivery. Recent Patents on Drug Delivery and Formulation. 2007;1(3):222-9.

31. Amidon S, Brown JE, Dave VS. Colon-targeted oral drug delivery systems: Design trends and approaches. AAPS Pharm Sci Tech. 2015;16(4):731-41.

32. Belali N, Wathoni N, Muchtaridi M. Advances in orally targeted drug delivery to colon. Journal of Advanced Pharmaceutical Technology and Research. 2019;10(3):100-6.

33. Rashid M, Kaur V, Hallan SS, Sharma S, Mishra N. Microparticles as controlled drug delivery carrier for the treatment of ulcerative colitis: A brief review. Saudi Pharm J. 2016;24(4):458-72. 


\section{PICTORIAL ABSTRACT}

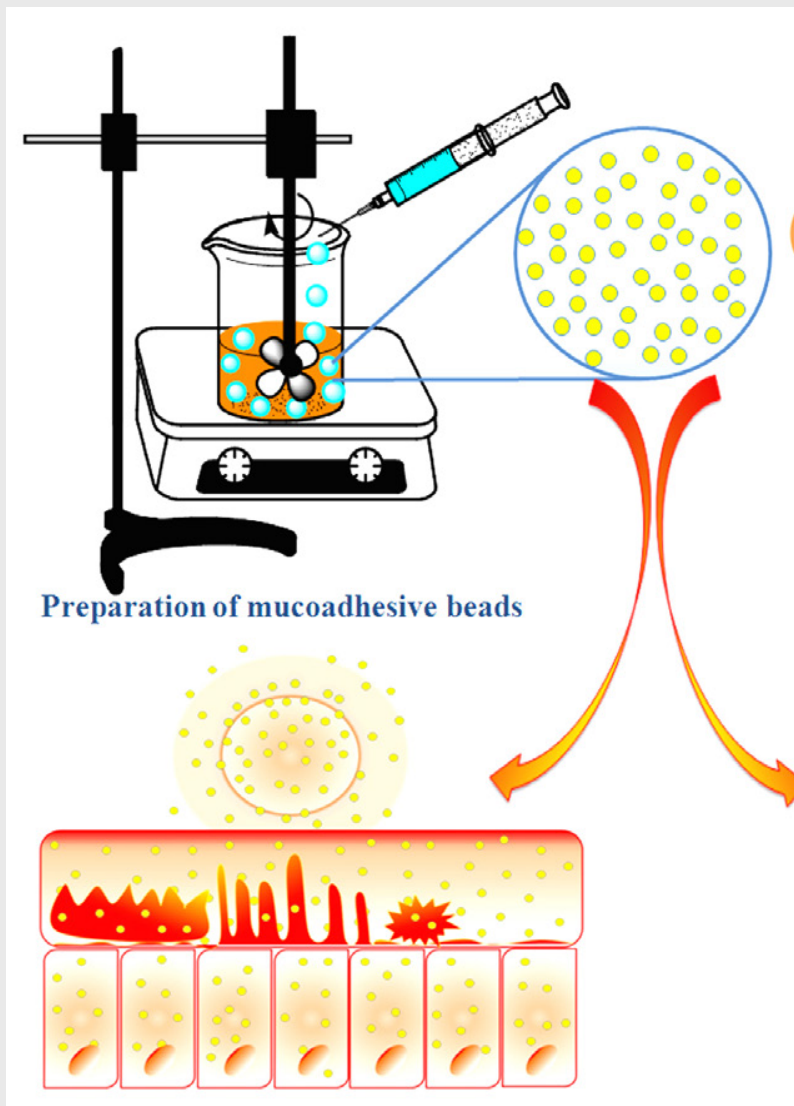

Delivery of mesalamine loaded beads to colon

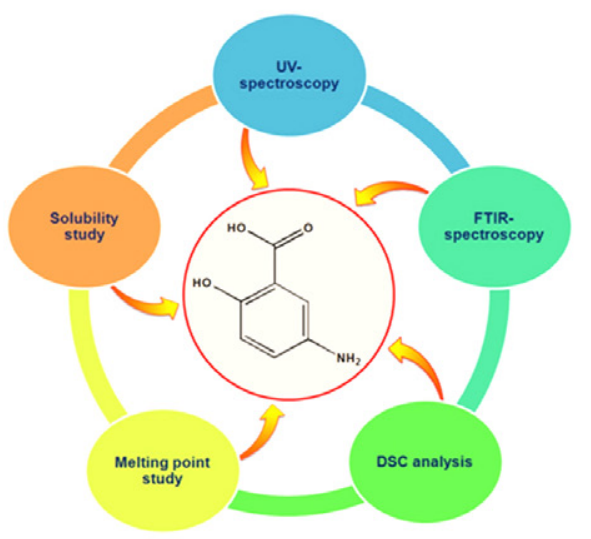

Preformulation studies of drug
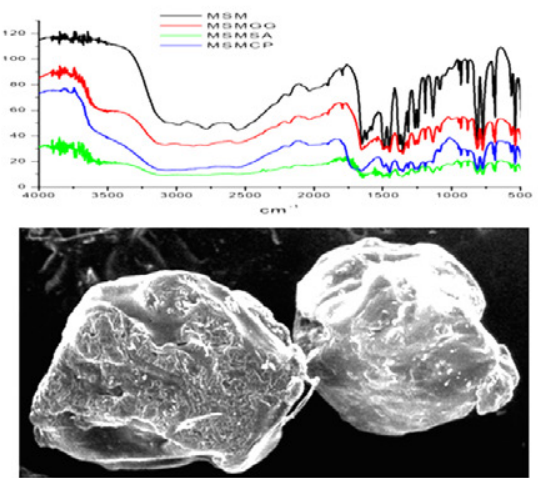

Characterization of beads

\section{SUMMARY}

- Inflammatory bowel disease is a disease of large intestine with clinical symptoms like abdominal pain, bloody diarrhea, weight loss and when untreated leads to fistulae, extreme abdominal pain, colon cancer, bowel function impairment and disability

- Mesalamine is a first line drug use for the treatment but it has low water solubility and low permeability thus low bioavailability in the cells. This problem can be overcome by targeted oral drug delivery system with mucoadhesive approach.

- Present study was aimed to assay physiochemical properties of mesalamine and to develop mesalamine loaded mucoadhesive beads using sodium alginate and guar gum and carbopol as a matrix polymer.

- The beads were prepared by ion gelation technique using $5 \%$ acidified $\mathrm{CaCl}_{2}$ solution. Total 5 different batches of formulation (MA-F1 to MA-F5) were prepared and coated with a Eudragit S 100. The beads were subjected for various characterization and evaluation.

- The melting point and $\lambda_{\max }$ of mesalamine was found to be $282^{\circ} \mathrm{C}$ and $220 \mathrm{~nm}$ respectively. DSC study gives a sharp endothermic peak at $282^{\circ} \mathrm{C}$ was similar to MP of drug and FTIR study confirms the drug and polymers were compatible.

- The Particle size, Zeta potential, \%EE, \%Yield, \%DL of optimized formulation (MA-F4) have was found to be $445.6 \pm 67.1 \mu \mathrm{m},-28.01 \pm 0.16 \mathrm{mV}, 94.98 \pm 3.22,96.27 \pm 3.22$ and $30.4 \pm 0.9$ respectively. The in vitro drug release was found to be $95.07 \pm 3.85$ at simulated gastric fluid $(\mathrm{pH} 7.4)$ in a sustained manner for longer periods (24 h).

- The study demonstrated that the prepared beads release the mesalamine in sustained release manner and helps in management of IBD. It can be good alternative to traditional delivery system which has drawback of high dose and more side effects. 


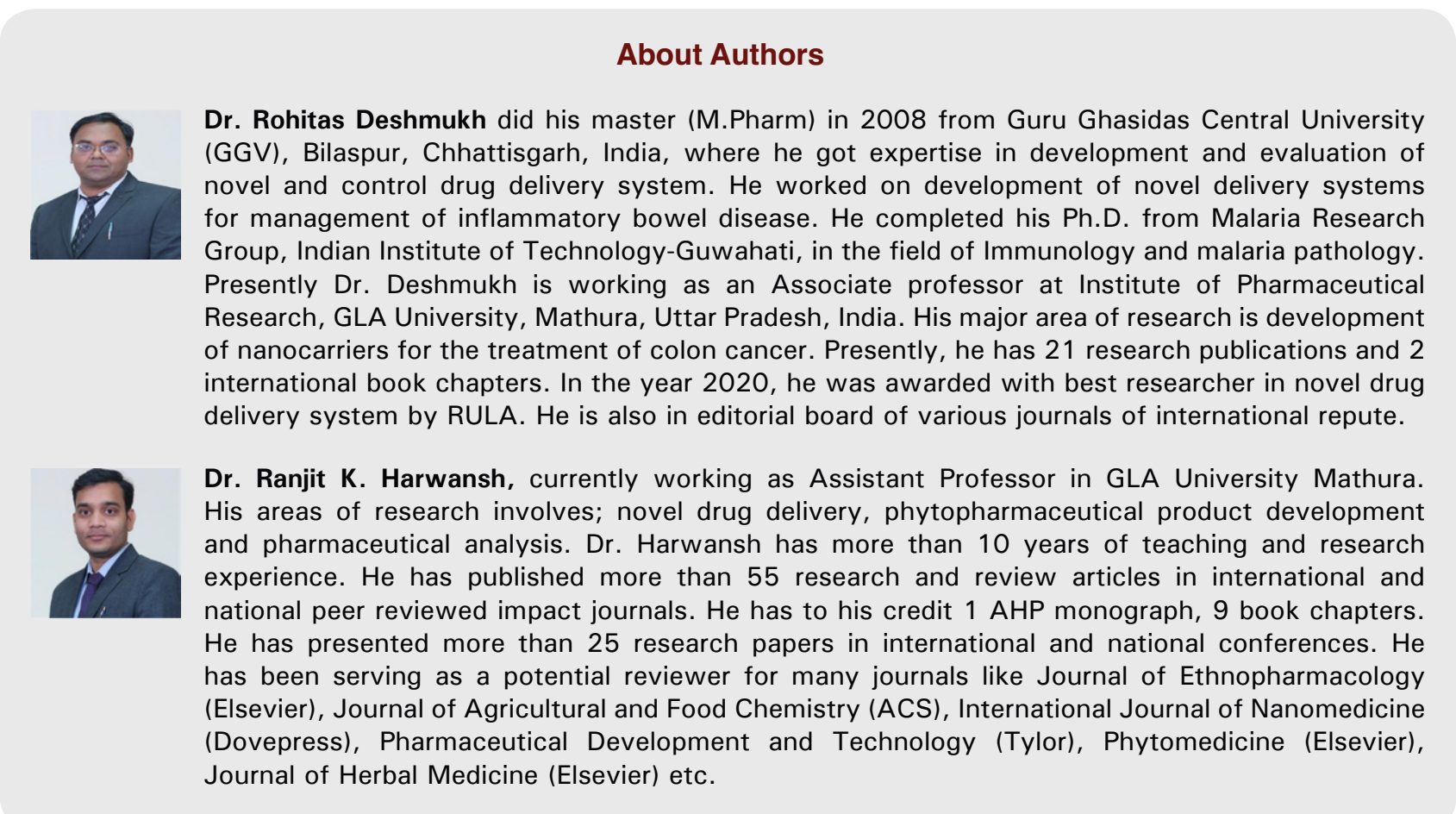

Cite this article: Deshmukh R, Harwansh RK. Preformulation Considerations Development and Evaluation of Mesalamine Loaded Polysaccharide-Based Complex Mucoadhesive Beads for Colon Targeting. Indian J of Pharmaceutical Education and Research. 2021;55(1):95-106. 